\title{
HYPERBOLIC KNOT COMPLEMENTS WITHOUT CLOSED EMBEDDED TOTALLY GEODESIC SURFACES
}

\author{
KAZUHIRO ICHIHARA and MAKOTO OZAWA
}

(Received 11 June 1999; revised 17 December 1999)

Communicated by J. A. Hillman

\begin{abstract}
It is conjectured that a hyperbolic knot complement does not contain a closed embedded totally geodesic surface. In this paper, we show that there are no such surfaces in the complements of hyperbolic 3-bridge knots and double torus knots. Some topological criteria for a closed essential surface failing to be totally geodesic are given. Roughly speaking, sufficiently 'complicated' surfaces cannot be totally geodesic.
\end{abstract}

2000 Mathematics subject classification: primary 57M25, 57M50.

Keywords and phrases: accidental peripheral, double torus, totally geodesic surface.

\section{Introduction}

The following conjecture was proposed by Menasco and Reid in [9].

CONJECTURE 1.1 ([9, Conjecture 1], [5, Problem 1.76]). A hyperbolic knot in the 3-sphere does not have a closed, totally geodesic surface embedded in its complement.

We remark that there are cusped hyperbolic 3-manifolds containing a closed embedded totally geodesic surface. Thus, it seems that Conjecture 1.1 applies only to 'simple' 3-manifolds like the 3-sphere. This conjecture was solved for alternating knots [7], almost alternating knots [2], toroidally alternating knots [1], closed 3-braids [6], Montesinos knots [10] and tunnel number one knots [9]. In fact, except for tunnel number one knots, any closed incompressible surface in these knot complements is meridionally compressible. This implies that there exists an accidental parabolic element, and so the surface is not even quasi-Fuchsian. For tunnel number one knots, the conjecture was solved algebraically in [9].

(c) 2000 Australian Mathematical Society 0263-61 15/2000\$A2.00+0.00 
In this paper we give another topological settlement of the conjecture for tunnel number one knot and prove the following result.

THEOREM 1.2. Hyperbolic 3-bridge knots and double torus knots do not have a closed totally geodesic surface embedded in their complements.

Moreover, we give slightly stronger results for free genus one knots. We also give two topological criteria for a closed essential surface failing to be totally geodesic in the complement of a hyperbolic knot $K$. Roughly speaking, sufficiently 'complicated' surfaces cannot be totally geodesic.

For a closed surface $S$ in $S^{3}-K$, we define the $\operatorname{order} o(S ; K)$ of $S$ for $K$ as follows [11]. Let $i_{*}: H_{1}(S) \rightarrow H_{1}\left(S^{3}-K\right)$ be the homomorphism induced by the inclusion map $i: S \rightarrow S^{3}-K$. Since $\operatorname{Im}\left(i_{*}\right)$ is a subgroup of $H_{1}\left(S^{3}-K\right)=\mathbb{Z}\langle$ meridian $\rangle$, there is an integer $m$ such that $\operatorname{Im}\left(i_{*}\right)=m \mathbb{Z}$. Then we define $o(S ; K)=|m|$.

THEOREM 1.3. Let $K$ be a hyperbolic knot in $S^{3}$ of genus $g, S$ a closed essential surface in $S^{3}-K$ having order $o$. If $o>3 g-3$, then $S$ is not totally geodesic.

The canonical genus $g_{c}(K)$ of $K$ is defined to be the minimum among genera of Seifert surfaces built by Seifert's algorithm on all diagrams of $K$. Let $V_{1}$ denote the volume of a regular ideal tetrahedron in $\mathbb{H}^{3}$ and $V_{g^{\prime}}$ that of a regular truncated tetrahedron with dihedral angles $\pi / 3 g^{\prime}$ in $\mathbb{A D}^{3}$.

THEOREM 1.4. Let $K$ be a hyperbolic knot in $S^{3}$ of canonical genus $g, S$ a closed essential surface of genus $g^{\prime}$ in $S^{3}-K$. If $g^{\prime} V_{g^{\prime}} \geq 60 g V_{1}$, then $S$ is not totally geodesic.

\section{Partial settlements}

Throughout this paper, we denote by $K$ a knot in $S^{3}$. We always denote the regular neighbourhood of $K$ by $N(K)$ and the exterior of $K$, which is the space obtained by removing the interior of $N(K)$ from $S^{3}$, by $E(K)$.

We start with giving some definitions. Let $S$ be a closed essential surface in $E(K)$. A nontrivial simple closed curve $l$ on $S$ is called an accidental peripheral when there is an annulus $A$ in $E(K)$ such that $A \cap S=\partial A \cap S=l$ and $A \cap \partial N(K)=\partial A-l$. We call such an essential surface $S$ with an accidental peripheral an accidental surface. Let $M_{1}$ and $M_{2}$ denote the closure of components of $S^{3}-S$, where $M_{1}$ contains $K$. If $S$ is accidental, then we can construct an essential annulus $A_{1}$ in $M_{1}-K$ by pasting two copies of an accidental annulus $A$ for $S$ and an annulus $\partial N(K)-A$. But the converse does not hold generally. An example is given in [3, Figure 2]. The following 
gives us a topological necessary condition for a closed essential surface isotopic to a totally geodesic surface.

LEMMA 2.1. If $K$ is hyperbolic and $S$ is totally geodesic in $S^{3}-K$, then $S$ is not accidental and neither $M_{1}-K$ nor $M_{2}$ contains an essential annulus.

REMARK. This lemma is well known, but we give a proof for the convenience of the reader. We remark that this necessary condition is rather far from complete, see [3].

PROOF. Suppose that $S$ is totally geodesic. Then the representation of $\pi_{1}(S)$ induced by a faithful discrete representation of $\pi_{1}\left(S^{3}-K\right)$ is Fuchsian. Therefore, it does not contain an accidental parabolic element. Assume $M_{1}-K$ or $M_{2}$ contains an essential annulus. Then its double contains an essential torus. However, when $S$ is totally geodesic, both doubles of $M_{1}-K$ and $M_{2}$ are hyperbolic, and hence atoroidal. It is a contradiction.

For tunnel number one knots, Conjecture 1.1 was solved in [9, Corollary 4$]$ by an algebraic method. The next theorem gives us a purely topological proof.

THEOREM 2.2. If $K$ is a tunnel number one knot in $S^{3}$, $S$ a closed essential surface in $E(K), M_{1}$ and $M_{2}$ the closure of components of $S^{3}-S$, where $M_{1}$ contains $K$, then $S$ is accidental or $M_{2}$ contains an essential annulus.

PROOF. Let $V_{1} \cup V_{2}$ be a genus two Heegaard splitting of $E(K)$, where $V_{1}$ is a compression body and $V_{2}$ is a handlebody. By an isotopy of $S$, we may assume that $S \cap V_{1}$ consists of mutually parallel essential separating disks and non-separating disks in $V_{1}$, where the labels are consecutive in $V_{1}$. Suppose that $\left|S \cap V_{1}\right|$ is minimal among all surfaces isotopic to $S$. Note that $\left|S \cap V_{1}\right| \neq 0$ because of the incompressibility of $S$. If $S \cap V_{2}$ is a single disk, then $S$ is a 2 -sphere and hence compressible. Otherwise, since $S \cap V_{2}$ is $\partial$-compressible in $V_{2}$, a $\partial$-compression of $S \cap V_{2}$ yields a band $b$ in $V_{1}$. By the minimality of $\left|S \cap V_{1}\right|, b$ forms an incompressible non- $\partial$-parallel annulus $A$ in $V_{1}$ together with some disk of $S \cap V_{1}$. If $A$ is non-separating in $V_{1}$, then there is an accidental annulus for $A$ in $V_{1}$. If $A$ is separating in $V_{1}$, then either there is an accidental annulus for $A$ in $V_{1}$, or a sub-annulus $A_{0}$ of $\partial V_{1}-A$ gives an essential annulus in $M_{1}$ or $M_{2}$. In particular, if $A_{0}$ is in $M_{1}$, then the next $\partial$-compression of $S \cap V_{2}$ yields a separating annulus parallel to $A$, and there is an essential annulus in $M_{2}$.

It follows from Lemma 2.1 and Theorem 2.3 that hyperbolic 3-bridge knot complements do not contain a closed embedded totally geodesic surface. 
THEOREM 2.3. Let $K$ be a 3-bridge knot in $S^{3}, S$ a closed essential surface in $E(K), M_{1}$ and $M_{2}$ the closure of components of $S^{3}-S$, where $M_{1}$ contains $K$. Then $S$ is meridionally compressible or $M_{2}$ contains an essential annulus.

PROOF. Suppose that $S$ is meridionally incompressible. Let $\left(B_{1}, T_{1}\right) \cup\left(B_{2}, T_{2}\right)$ be a 3-bridge tangle decomposition of $\left(S^{3}, K\right), D_{i}(i=1,2)$ a disjoint union of disks in $B_{i}$ which are co-bounded by the strings of $T_{i}$ and arcs in $\partial B_{i}$. By an isotopy of $S$, we may assume that $S \cap D_{1}=\emptyset, S \cap B_{1}$ consists of essential disks in $B_{1}-T_{1}$, $S \cap D_{2}$ consists of essential arcs on $S \cap B_{2}$, and $\left(S \cap B_{2}\right)-D_{2}$ consists of open disks. Under the above conditions, we assume that $\left|S \cap B_{1}\right|$ is minimal among all surfaces isotopic to $S$. By the incompressibility of $S,\left|S \cap B_{1}\right| \geq 1$. We perform a a-compression of $S \cap B_{2}$ along an outermost disk in $D_{2}$, and obtain an incompressible annulus $A_{1}$ in $B_{1}-T_{1}$ which consists of a disk of $S \cap B_{1}$ and a resultant band $b$. It follows from the meridional incompressibility of $S$ that $S \cap B_{1}$ consists of mutually parallel disks which divide $\left(B_{1}, T_{1}\right)$ into the trivial 1-string tangle $\left(B_{11}, T_{11}\right)$ and the trivial 2-string tangle $\left(B_{22}, T_{22}\right)$, and the band $b$ runs over $\left(B_{22}, T_{22}\right)$. Note that $\left|S \cap B_{1}\right|$ is an even integer greater than 1 since $S$ separates $S^{3}$ into $M_{1}(\supset K)$ and $M_{2}$. If we perform the $\partial$-compression of $S \cap B_{2}$ along the next outermost arc, then we obtain an incompressible annulus $A_{2}$ in $B_{1}-T_{1}$ which is parallel to $A$. Hence, there exists an annulus $A_{0}$ in $B_{1}-T_{1}$ connecting $A_{1}$ and $A_{2}$. We note that $A_{0}$ is contained in $M_{2}$. If $A_{0}$ is $\partial$-parallel in $M_{2}$, then it contradicts the minimality of $\left|S \cap B_{1}\right|$. Since $S \cap D_{2}$ are essential arcs in $S \cap B_{2}, A_{i}(i=1,2)$ is incompressible in $M_{2}$. Therefore, $A_{0}$ is incompressible in $M_{2}$, thus $A_{0}$ is an essential annulus in $M_{2}$.

A knot $K$ in $S^{3}$ is said to be a double torus if $K$ is contained in a genus two Heegaard surface of $S^{3}$. It is known that 2-bridge knots, pretzel knots, tunnel number one knots, and free genus one knots are double torus. Combined with previous results, the next theorem completes the proof of Theorem 1.2.

THEOREM 2.4. Let $K$ be a non-cable double torus knot with respect to a genus two Heegaard splitting $\left(F ; V_{1}, V_{2}\right)$ of $S^{3}, S$ a closed essential surface in $E(K)$. Then $S$ is accidental or $M_{1}-K$ or $M_{2}$ contains an essential annulus.

REMARK. The condition 'non-cable' cannot be omitted. It is easy to see that cable knots with tunnel number one companion knots are double torus. In general, an accidental surface for the companion knot is not accidental for a cable knot if it is disjoint from its companion solid torus.

PROOF. We divide the proof into two cases, whether $F-K$ is incompressible in $S^{3}-K$ or not. 
Suppose that $F-K$ is compressible in $S^{3}-K$ and let $D$ be a compressing disk for $F-K$ in $S^{3}-K$. We may assume that by exchanging it if necessary, $D$ is a non-separating disk in $V_{1}$. Put $V_{1}^{\prime}=c l\left(V_{1}-N(D)\right)$ and $F^{\prime}=\partial V_{1}^{\prime}$. Then $V_{1}^{\prime}$ is a solid torus and its core, say $\gamma$, is a tunnel number one knot. Therefore, $K$ is a tunnel number one knot or a cable knot with a companion knot $\gamma$. In the former case, Theorem 2.3 gives the conclusion. In the latter case, if $\gamma$ is non-trivial, then this contradicts the assumption of $K$. Hence $\gamma$ is trivial and $K$ is a torus knot. Since any incompressible closed surface in a torus knot complement must be isotopic to a peripheral torus, this case does not occur.

Next, suppose that $F-K$ is incompressible in $S^{3}-K$, and assume that $|S \cap F|$ is minimal up to isotopy of $S$. Then each loop of $S \cap F$ is essential in both $S$ and $F-K$. If there are mutually parallel loops of $S \cap F$ in $F$ or there is a loop of $S \cap F$ which is parallel to $K$ in $F$, then $M_{i}$ contains an essential annulus or $S$ is accidental. Otherwise, $S \cap F$ consists of a single loop which separates $F$ into two once punctured tori $F_{i}\left(\subset M_{i}\right)$, and $K$ is non-separating in $F_{i}$. Since the loop $S \cap F$ is essential in $S$, $F_{1}$ is incompressible in $M_{1}$. Let $D$ be a compressing disk for $S$ in $S^{3}$, hence in $M_{1}$. We may assume that by modifying $D$ if necessary, $D \cap F_{1}$ consists of essential arcs in $F_{1}$. Note that by the incompressibility of $S$ in $S^{3}-K, D \cap F_{1} \neq \emptyset$. Let $\alpha$ be an outermost arc of $D \cap F_{1}$ in $D$, and let $\delta$ be the corresponding outermost disk in $D$. We perform a $\partial$-compression of $F_{1}$ along $\delta$ in $M_{1}$, and get an annulus $F_{1}^{\prime}$ disjoint from $F_{1}$. Since $F_{1}$ is incompressible in $M_{1}, F_{1}^{\prime}$ is also incompressible in $M_{1}$, hence in $M_{1}-K$. If $F_{1}^{\prime}$ is essential in $M_{1}-K$, then we have the desired conclusion. So, suppose that $F_{1}^{\prime}$ is parallel to a sub-annulus $A_{1}$ in $\partial M_{1}$, and let $V$ be the solid torus co-bounded by $F_{1}^{\prime}$ and $A_{1}$. Now, to recover $F_{1}$, we attach a band $b$ corresponding to the $\partial$-compressing disk $\delta$, to $F_{1}^{\prime}$. If $b$ is contained in $V$, then we can find a compressing disk for $F_{1}$ in $M_{1}$, a contradiction. Otherwise, $F_{1}$ is parallel to a sub-surface in $\partial M_{1}$, thus in $S$. This implies that $K$ is isotopic to a loop in $S$, hence $S$ is accidental.

A Seifert surface $F$ for $K$ in $S^{3}$ is said to be free if the fundamental group $\pi_{1}\left(S^{3}-F\right)$ is free. We define the free genus of $K$ as the minimal genus over all free Seifert surfaces for $K$. For free genus one knots, we also obtain the following theorem.

THEOREM 2.5. If $K$ is a free genus one knot in $S^{3}, S$ a closed essential surface in $E(K), M_{1}$ and $M_{2}$ the closure of components of $S^{3}-S$, where $M_{1}$ contains $K$, then $S$ is accidental or $M_{2}$ contains an essential annulus.

PROOF. Let $F$ be a genus one free Seifert surface for $K$. We may assume that $F \cap S$ consists of essential loops in both $F$ and $S$, and assume that $|F \cap S|$ is minimal. Since $\pi_{1}\left(S^{3}-F\right)$ is a free group, $F \cap S \neq \emptyset$. If there is a loop of $F \cap S$ which is parallel to $K$ in $F$, then $S$ is accidental. Otherwise, all loops of $F \cap S$ are non-separating and mutually parallel in $F$. Since $S$ separates $S^{3}$, hence $F$, there is an annulus component 
$A$ of $F-S$ in $M_{2}$. Then, the minimality of $|F \cap S|$ assures us that $A$ is essential in $M_{2}$.

\section{Criteria for failing to be totally geodesic}

Theorem 1.3 follows from the next theorem and Lemma 2.1 .

THEOREM 3.1. Let $K$ be a knot in $S^{3}$ of genus $g$, $S$ a closed essential surface in $E(K)$ having order $o$. If $o>3 g-3$, then $S$ is accidental or $M_{1}-K$ or $M_{2}$ contains an essential annulus.

PROOF. Suppose that $o>3 g-3$ holds. Let $F$ be a minimal genus Seifert surface for $K$. We assume that $|S \cap F|$ is minimal among all minimal genus Seifert surfaces. Then each curve of $S \cap F$ is essential in both $S$ and $F$.

Let us show that $|S \cap F| \geq o$. To do this, we construct a finite connected graph, say $G_{S}$, by identifying a connected component of $S-(S \cap F)$ with a vertex, and a curve of $S \cap F$ with an edge. We fix an orientation of each edge of $G_{s}$ induced from orientations of $S, F$ and $S^{3}$. If $G_{S}$ is a tree, then each edge is a cutting edge, and so each curve of $S \cap F$ is separating on $S$. But this contradicts the fact that $o>0$. Hence $G_{S}$ has at least one cycle. Let $c$ be a cycle of $G_{S}$ with an arbitrary orientation. We give the weight +1 to an edge of $c$ when the orientation of the edge coincides with that of $c$, and -1 otherwise. Then $|S \cap F|=\left|E\left(G_{S}\right)\right| \geq|o(c)|$ holds, where $o(c)$ is the sum of the weights of edges of $c$. On the other hand, a loop on $S$ can be constructed for $c$ such that the linking number of it and $K$ is equal to $o(c)$. This implies that $|o(c)| \geq o$, and hence $|S \cap F| \geq 0$.

By the above argument and the assumption, $|S \cap F| \geq 0>3 g-3$ holds. In the case that $n:=|S \cap F|>3 g-2$, we find an accidental annulus for $S$ or a sub-annulus bounded by two curves of $S \cap F$ on $F$, since there are at most $3 g-2$ mutually non-parallel and non- $\partial$-parallel simple closed curves on $F$. This sub-annulus is essential in $M_{i}$, since otherwise, we can reduce $|S \cap F|$. In the remaining case, that is, $n=3 g-2$, we consider the graph $G_{F}$ constructed from $F$ in the same way as $G_{S}$. Suppose that the curves of $S \cap F$ are mutually non-parallel and non- $\partial$-parallel on $F$. Then that curves give a pants decomposition of $F$. This decomposition admits a checkerboard-coloring, since each pair of pants is contained in $M_{1}$ or $M_{2}$. Let us note that $G_{F}$ has the following properties:

(1) There is a vertex $v_{0} \in V\left(G_{F}\right)$ having degree 2;

(2) Every $v \in V\left(G_{F}\right)-\left\{v_{0}\right\}$ has degree 3 .

Then the next lemma gives us a contradiction. Consequently, $S \cap F$ has some mutually parallel curves or a $\partial$-parallel curve in $F$, and hence we can find an accidental annulus for $S$ or an essential annulus in $M_{i}$. 
LEMMA 3.2. Let $G$ be a finite graph satisfying the following two properties:

(1) There is a vertex $v_{0} \in V(G)$ having degree 2;

(2) Every $v \in V(G)-\left\{v_{0}\right\}$ has degree 3.

Then $G$ is not a bipartite graph.

ProOF. Suppose that there is a 2-coloring $f: V(G) \rightarrow\{B, W\}$, and assume that $f\left(v_{0}\right)=B$. Let $V_{w}$ be the set $\{v \in V(G) \mid f(v)=W\}$ and let $V_{b}$ be the set $\{v \in V(G) \mid f(v)=B\}$. Then the number of edges which are incident to the vertices of $V_{b}$ is $3\left(\left|V_{b}\right|-1\right)+2$, and the number of those for $V_{w}$ is $3\left|V_{w}\right|$. However, since every edge of $G$ connects a vertex of $V_{b}$ and one of $V_{w}$, these values must be equal. Hence a contradiction.

REMARK. In the proof of Theorem 3.1, we note that $|S \cap F|=o>0$ if and only if $S \cap F$ consists of mutually parallel non-separating loops in $S$.

Finally we prove Theorem 1.4.

PROOF OF THEOREM 1.4. On one hand, it was shown in [4] that the volume of the complement of a hyperbolic knot is less than $120 \mathrm{~g} V_{1}$, where $g$ is the canonical genus of the knot and $V_{1}$ is the volume of a regular ideal tetrahedron in $\mathbb{H}^{3}$. On the other hand, it was shown in [8] that the minimal volume of complete hyperbolic 3-manifolds with a totally geodesic boundary of genus $g^{\prime}$ is $g^{\prime} V_{g^{\prime}}$, where $V_{g^{\prime}}$ is the volume of a regular truncated tetrahedron with dihedral angles $\pi / 3 g^{\prime}$ in $\mathbb{H}^{3}$. Recall that $K$ is a hyperbolic knot in $S^{3}$ of canonical genus $g$ and $S$ is a closed essential surface of genus $g^{\prime}$ in $E(K)$. Now, suppose that $S$ is totally geodesic in $S^{3}-K$. Let $M_{1}$ and $M_{2}$ be the closure of components of $S^{3}-S$, where $M_{1}$ contains $K$. Then both $M_{1}-K$ and $M_{2}$ are hyperbolic 3-manifolds with totally geodesic boundary, and so $\operatorname{vol}\left(S^{3}-K\right)=\operatorname{vol}\left(M_{1}-K\right)+\operatorname{vol}\left(M_{2}\right)$. As a consequence, we get $60 g V_{1}>g^{\prime} V_{g^{\prime}}$.

REMARK. Since $V_{1}$ is approximately 1.01494 and $V_{g^{\prime}}$ is computed by using the formula given in [8], we can see that the inequality $60 g V_{1}>g^{\prime} V_{g^{\prime}}$ fails if $g=1$ and $g^{\prime}>16$. Therefore, there is no closed embedded totally geodesic surface of genus $g^{\prime}>16$ in a hyperbolic knot complement of canonical genus one. Note that $V_{g^{\prime}} \nearrow V_{0}$ as $g^{\prime} \rightarrow \infty$, where $V_{0}$ is the volume of a regular ideal octahedron in $\mathbb{H}^{3}$ and is approximately 3.6639 .

\section{References}

[1] C. Adams, 'Toroidally alternating knots and links', Topology 33 (1994), 353-369. 
[2] C. Adams, J. Brock, J. Bugbee, T. Comar, K. Faigin, A. Huston, A. Joseph and D. Pesikoff, 'Almost alternating links', Topology Appl. 46 (1992), 151-165.

[3] C. Adams and A. Reid, 'Quasi-Fuchsian surfaces in hyperbolic knot complements', J. Austral. Math. Soc. (Series A) 55 (1993), 116-131.

[4] M. Brittenham, 'Bounding canonical genus bounds volume', preprint available on http://xxx.lanl.gov/abs/math.GT/9809142.

[5] R. Kirby, 'Problems in low-dimensional topology', in: Geometric topology, Part 2 (ed. W. H. Kazez), Studies in Adv. Math. (Amer. Math. Soc., Inter. Press, 1997).

[6] M. T. Lozano and J. H. Przytycki, 'Incompressible surfaces in the exterior of a closed 3-braid $I$, surfaces with horizontal boundary components', Math. Proc. Cambridge Philos. Soc. 98 (1985), 275-299.

[7] W. Menasco, 'Closed incompressible surfaces in alternating knot and link complements', Topology 23 (1984), 37-44.

[8] Y. Miyamoto, 'Volumes of hyperbolic manifolds with geodesic boundary', Topology 33 (1994), 613-629.

[9] W. Menasco and A. Reid, 'Totally geodesic surfaces in hyperbolic link complements', in: Topology '90 (eds. B. Apanasov, W. Neumann, A. Reid and L. Siebenmann) (de Gruyter, Amsterdam, 1992).

[10] U. Oertel, 'Closed incompressible surfaces in complements of star links', Pacific J. Math. 111 (1984), 209-230.

[11] M. Ozawa, 'Synchronism of an incompressible non-free Seifert surface for a knot and an algebraically split closed surface in the knot complement', to appear in Proc. Amer. Math. Soc.

Department of Mathematics

Tokyo Institute of Technology

Ookayama 2-12-1, Meguroku

Tokyo 152-8551

Japan

e-mail: ichihara@math.titech.ac.jp
Department of Mathematics

School of Education

Waseda University

Nishiwaseda 1-6-1, Shinjuku-ku

Tokyo 169-8050

Japan

e-mail: ozawa@mn.waseda.ac.jp 